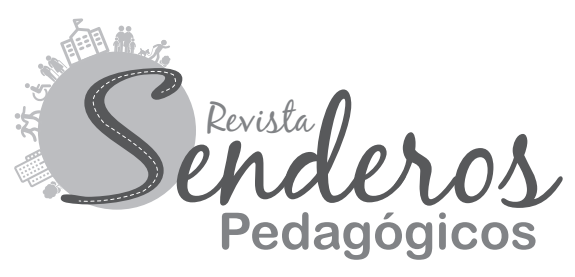

\title{
La importancia de la estimulación adecuada durante el neurodesarrollo en la primera infancia ${ }^{1}$
}

\author{
The Importance of Adequate Stimulation during \\ Neurodesuring in Early Childhood
}

\author{
Autora: \\ Mónica Alejandra Gallego Marín
M \\ Recibido: 20/06/2019 \\ Aprobado: 22/11/2019
}

1. El presente artículo se deriva de la investigación titulada: Aportes de la neuroeducación a la educación inicial de niños entre los o y 2 años de edad. Un abordaje sociocrítico de las prácticas pedagógicas en las Ludotekas de Medellín, la cual hace parte de la Maestría en Educación Línea Cognición y Creatividad de la Universidad de Antioquia. Investigador principal Mónica Alejandra Gallego Marín.

2. Licenciada en Educación Especial; Especialista en Neurodesarrollo y Aprendizaje; estudiante de último semestre de la Maestría en Educación línea Cognición y Creatividad. alejandragm425@gmail.com Creatividad alejondragnats

\section{Resumen}

El artículo analiza los principales estudios sobre estimulación adecuada y neurodesarrollo, e identifica la importancia de estos en la primera infancia. Se justifica que la estimulación en los primeros años de vida es un reto para docentes y profesionales que trabajan con primera infancia, dado que son ellos quienes proporcionan los medios para que el entorno aporte significativamente al desarrollo de los niños; así mismo, son quienes pueden detectar tempranamente factores de riesgo que puedan desviar el curso del mismo. Se realizó una revisión sistemática de 52 estudios en bases de datos científicas como Scielo, Dialnet, Redalyc, Springer y buscadores especializados como: Google académico y PubMed. Se concluye que en el desarrollo de los niños se presentan avances y retrocesos que van a depender de la interacción entre genética y ambiente; se demuestra que la estimulación brinda múltiples 
ventajas para que este desarrollo se de en las mejores condiciones desde el neurodesarrollo.

Palabras clave: estimulación adecuada, neurodesarrollo, primera infancia.

\begin{abstract}
The article analyzes the main studies on adequate stimulation and neurodevelopment, identifying the importance of these in early childhood. It is justified that the stimulation in the first years of life is a challenge for teachers and professionals working with early childhood, since they are the ones who provide the means for the environment to significantly contribute
\end{abstract}

to the development of children; likewise, they are the ones who can detect early risk factors that can deviate the course of the developement path. We conducted a systematic review of 52 studies in scientific databases such as Scielo, Dialnet, Redalyc, Springer and specialized search engines such as Google academic and PubMed. It is concluded that in child developement there are advances and setbacks that will depend on the interaction between genetics and environment, demonstrating that stimulation offers multiple advantages so that it is in the best conditions from neurodevelopment.

Keywords: Proper stimulation, neurodevelopment, early childhood.

\section{Introducción}

Históricamente el término desarrollo se ha referido a los cambios físicos observables en tamaño o estructura de un organismo durante un periodo determinado; concepción que ha sufrido cambios significativos con el paso de los años; por su parte, los hallazgos de diversas investigaciones en disciplinas como: neurociencias, ciencias neurobiológicas, comportamentales o sociales han llevado a comprender que ese concepto de desarrollo secuencial y acumulativo no es el adecuado; por tal motivo, se hace necesaria una reformulación que contemple que el desempeño de un mismo niño es variable a través del tiempo, y que las producciones son cambiantes en cada niño, incluso si son de la misma edad, esto "da cuenta de sus avances y retrocesos y de aquellos momentos en que coexisten viejas y nuevas concepciones” (MEN, 2009, p. 16).

Actualmente, el desarrollo infantil es concebido como un proceso que no es lineal, sino que se caracteriza por un funcionamiento irregular de avances y retrocesos, que tiene un sustento biológico, psicológico y social. Shonkoff y Garner (2012) y Martins y Ramallo (2015) afirman que en los primeros años de vida se van configurando las habilidades que posibilitarán una adecuada interacción del niño con su entorno social; durante esta etapa se forma la arquitectura del 
cerebro, a partir de la interacción entre la genética y el ambiente en el que vive el niño. El cerebro del niño se encuentra en un desarrollo continuo, sujeto a modificaciones de acuerdo con la estimulación que el ambiente le proporcione. El neurodesarrollo "puede verse como un proceso en el cual el niño participa junto a su medio ambiente, influyéndose mutuamente y evolucionando en una dirección particular" (Ponce, 2017, p. 408); en este sentido, es importante tener en cuenta no solo al niño, sino también las interacciones entre él, sus padres o cuidadores, familia y entorno inmediato.

La estimulación brinda múltiples ventajas al desarrollo de los niños, debido a que ellos asimilan toda la información que se encuentra en el ambiente, lo que permite que se den aprendizajes como "consecuencia de la interacción dinámica entre su potencial genético y las experiencias recibidas, hecho que determinará las posibilidades que pueda tener para enfrentar el futuro" (Alegría, 2008, p. 6).

Investigaciones como las de Figueiras, Neves, Ríos, y Benguigui (2011), y Pastor, Nashiki y Pérez (2010) resaltan la importancia de tener una observación constante del proceso de desarrollo en los diferentes contextos en los que el niño se desenvuelve; el ambiente en el cual está inmerso va a influenciar su desarrollo. El cuidado, la alimentación, la lactancia y la estimulación tendrán una incidencia directa en el crecimiento y desarrollo durante los primeros años de vida. Romero y Muñoz (2016) afirman que "la estimulación en edades tempranas incrementa las probabilidades de rendimiento positivo en diferentes ámbitos; asimismo, un desarrollo hipo estimulado -en el que las bases de estas capacidades son débilespuede significar un riesgo de presentar dificultades futuras" (p. 56).

La estimulación adecuada adquiere particular importancia durante el neurodesarrollo por tratarse de un período en el que se producen muchas más conexiones neuronales, mayor plasticidad cerebral, y es la etapa que se caracteriza por "el desarrollo de funciones básicas en el futuro del niño (afectividad, motricidad, lenguaje y desarrollo sensorial) por esto la estimulación constituye un aspecto importante para su futuro, dado que garantiza los primeros contactos con el medio exterior" (Huepp, 2005, p. 33).

Brindar una estimulación durante el neurodesarrollo en la primera infancia, va a permitir que de manera oportuna se detecten factores de riesgo biológico y se identifiquen "dificultades en el proceso de maduración; detección de lesiones y alteraciones del desarrollo, lo cual, puede orientar el pronóstico y seguimiento de las lesiones y sus secuelas a lo largo del tiempo" (Zuluaga, 2001 citado en Ávila, 2012, p. 92). 
La importancia de los primeros tres años de vida en el desarrollo de los niños ha sido reconocida a nivel mundial en temas de vigilancia sanitaria por la Organización Mundial de la Salud (OMS) (2007), la cual considera que el período de la primera infancia es la fase de desarrollo más importante de todo el ciclo vital. "Un desarrollo de la primera infancia (DPI) saludable que abarque los dominios físico, socioemocional y lingüístico-cognitivo del desarrollo, ejercerá una influencia notable sobre el bienestar durante toda la vida" (p. 7).

Investigaciones como las de Mustard, Young y Manrique (2003) y la de Martínez (2014), realizan una revisión frente al desarrollo infantil y concluyen que este tiene un efecto significativo sobre las alteraciones físicas y mentales futuras en la vida adulta; además agregan que el desarrollo corresponde a una interacción dinámica entre las características del niño y las influencias del medio.

La estimulación se ha identificado como un factor importante para la primera infancia. Los estudios actuales sugieren que la efectividad de esta es la plasticidad, conocida como la capacidad de aprendizaje y moldeamiento del cerebro durante los primeros años de vida (Sibaja, Sánchez, Rojas y Fornaguera, 2016, p. 142).

Algunos autores afirman que existe una relación bidireccional entre lo biológico y el ambiente como factores de múltiples variaciones que intervienen en el desarrollo y que se expresan a través de un proceso adaptativo; al respecto, Pinto (2008 citado en Chinome, Rodríguez y Parra, 2017) y Zuluaga (2001 citado en Chinome, Rodríguez y Parra, 2017) explican que las características de los genes del ser humano son sometidas a interacción con el medio ambiente, "lo cual induce a cambios negativos o positivos a nivel de neurodesarrollo, sobre todo en periodos sensibles como es el primer año de vida, ya que de allí pueden derivar anomalías permanentes sobre la estructura y función cerebral” (p. 3).

Los estudios revisados establecen que la falta o poca estimulación, puede afectar el proceso de neurodesarrollo en cuanto que impide la proliferación de las células nerviosas, lo que genera que decrezcan progresivamente a pesar de las condiciones favorables que tiene la corteza cerebral desde el momento del nacimiento. Por lo tanto, conocer la importancia de la estimulación adecuada en los primeros años de vida es un reto para docentes y profesionales que trabajan con primera infancia, dado que es el adulto quien proporciona los medios adecuados para que el entorno aporte significativamente en el desarrollo de los niños; así mismo, es quien puede detectar tempranamente factores de riesgo que puedan desviar el curso del mismo. 
En ese orden de ideas, y como resultado de la investigación titulada: Aportes de la neuroeducación a la educación inicial de niños entre los o y 2 años de edad: un abordaje sociocrítico de las prácticas pedagógicas en las Ludotekas de Medellín, se deriva el presente artículo con el objetivo de analizar los principales estudios sobre estimulación adecuada y neurodesarrollo e identificar la importancia de estos en la primera infancia.

\section{Método}

Se realizó una revisión sistemática de 52 estudios relacionados con estimulación adecuada, desarrollo, neurodesarrollo y primera infancia. Se utilizaron como documentos oficiales investigaciones, artículos derivados de investigaciones, artículos de revista, libros, informes de eventos académicos, tesis de maestría y de doctorado.

\section{Selección de los estudios y estrategia de búsqueda}

Los estudios de la presente revisión se identificaron a través de la búsqueda automatizada en bases de datos científicas como: Scielo, Dialnet, Redalyc, Springer y buscadores especializados como: Google académico y PubMed. Asimismo, se utilizaron repositorios institucionales de diferentes universidades y se consultaron las bases de datos ofrecidas por la Universidad de Antioquia, tales como: DOAJ, Ebsco, Apa Psyc Net.

Los descriptores utilizados para realizar la búsqueda y clasificación de los estudios y para lograr organizar y estructurar la revisión fueron: Desarrollo cerebral en primera infancia, Neurodesarrollo y Estimulación; Neurodesarrollo en Primera infancia; Estimulación y Primera infancia.

Para la búsqueda se utilizaron los siguientes métodos:

- Elaboración de una matriz bibliográfica, en la que se registraron todos los documentos encontrados.

- Selección de estudios, a partir de los siguientes criterios de inclusión: ser investigaciones, artículos, libros, informes o tesis originales, con título y autores expresamente citados y ser publicados en bases de datos confiables; adicional, que no superaran 10 años de haber sido realizados; sin embargo, algunos de ellos no fue posible encontrarlos bajo dicho criterio y son entre los años 2003 y 2008.

- Síntesis de la información, que hizo posible la organización y estructuración de la información. 
- Análisis de documentos, lo que permitió extraer información relevante y dividirla en las categorías emergentes, las cuales fueron: desarrollo, neurodesarrollo, estimulación adecuada y primera infancia.

\section{Resultados}

A continuación se relacionan las definiciones, conceptos y posturas de diferentes autores que en sus estudios se han interesado en las categorías emergentes del presente artículo como son: desarrollo, neurodesarrollo, estimulación adecuada y primera infancia.

\section{Desarrollo}

Una de las características de la primera infancia es el constante proceso evolutivo hacia la adultez, con el paso necesario por cada una de las etapas de crecimiento y desarrollo, en el que se adquieren habilidades como producto del proceso activo de interacción con el entorno. Tal proceso, se encuentra particularizado por la relación entre genética y ambiente.

Se entiende por desarrollo "el avance en la diferenciación de células y tejidos, lo que significa complejidad creciente de las estructuras orgánicas y funcionales; es, pues, la adquisición de nuevas funciones mediante la maduración” (Posada, Gómez y Ramírez, 2016, p.17).

El desarrollo se caracteriza por un funcionamiento irregular de avances y retrocesos, que tiene un sustento biológico, psicológico y social, es concebido como un proceso de reconstrucción y reorganización permanente, lo cual generó el surgimiento de nuevos hechos y teorías que permitieran estudiar y repensar las características de las etapas de desarrollo y los desempeños de los niños en cada una de ellas. Ante esto, el Ministerio de Educación Nacional (MEN) afirma que, si bien el desarrollo ocurre a lo largo del ciclo vital, "está demostrado que existen momentos particularmente sensibles para actuar en favor de la salud, la nutrición, el crecimiento, desarrollo y bienestar de los niños, los cuales deben ser considerados al identificar las acciones para emprender la atención integral” (MEN, 2013, p. 109).

Es necesario tener en cuenta diferentes puntos de vista sobre el desarrollo que faciliten una visión más integral del proceso evolutivo y que complementen la visión biológica tradicional. Las teorías del desarrollo que se han encargado de ello son las planteadas por: Arnold Gesell quien "dice que los genes son potentes y determinan consecuencias evolutivas inevitables en ambientes diferentes. El 
modelo se olvida de las experiencias personales y del ambiente cultural" (Alarcon y Ellies, 2007, p. 23); Sigmund Freud habla del impulso libidinal, que se origina en una estructura fisiológica en desequilibrio, principio de las motivaciones. Se concebía que el ambiente podía reprimir, diferenciar o reencauzar los impulsos y sublimarlos; Jean Piaget, realiza un "análisis del desarrollo cognoscitivo; Erik Erikson complementa la teoría psicoanalítica y hace énfasis en la visión social del proceso" (Posada, Gómez y Ramírez, 2016, p.17); Lev Vygotsky enfatiza en la influencia de los contextos sociales y culturales para la adquisición del conocimiento y, Urie Bronfenbrenner, comprende el estudio de la acomodación "entre un ser humano activo, en desarrollo, y las propiedades cambiantes de los entornos inmediatos en los que vive” (Alarcón y Ellies, 2007, p. 82).

En las actuales concepciones, el niño es el gestor de su propio desarrollo, el cual tiene influencias de carácter genético y ambiental; la interacción de estos dos factores determina el curso del desarrollo; Francisco (2011) afirma que las desventajas de ambos pueden llevar a un desarrollo inadecuado, dichas desventajas se conocen como factores de riesgo, que deben ser detectados por los adultos, padres, cuidadores, personal de la educación y de la salud, con el fin de intervenir y asegurar las mejores condiciones para el proceso de desarrollo. Los resultados de diversas investigaciones han demostrado que promover un adecuado desarrollo beneficia a los niños, las familias y las comunidades. Los programas de desarrollo infantil promueven niveles más elevados de habilidades sociales y emocionales, por lo tanto, representan una manera efectiva de fortalecer la sociedad, garantizando que cada individuo viva de acuerdo con sus potenciales máximos (OEA, 2005, p. 1). De acuerdo con el MEN, la concepción de desarrollo se caracteriza por tres aspectos fundamentales:

En primer lugar, el desarrollo cognitivo, lingüístico, social y afectivo de los niños no es un proceso lineal; por el contrario, se caracteriza por un funcionamiento irregular de avances y retrocesos. En segundo lugar, el desarrollo no tiene un principio definitivo y claro, es decir, no inicia desde cero. Y, en tercer lugar, el desarrollo no parece tener una etapa final, en otras palabras, nunca concluye, siempre podría continuar (MEN, 2009, p. 18).

Para garantizar la individualización y ser coherentes con las nuevas concepciones del desarrollo, es importante no establecer fronteras entre las edades del desarrollo infantil, "cada niño tiene su propio ritmo y algunas capacidades se van construyendo poco a poco, entre 'ires y venires'. Por lo tanto, establecer la relación entre una determinada edad y una determinada conducta no siempre resulta exitoso" (MEN, 2009, p. 12). 
El desarrollo en los primeros años de vida es una etapa crucial en la vida del ser humano, debido a que en ella se generan las bases para los aprendizajes posteriores, se perfila la arquitectura del cerebro y diseña el futuro comportamiento; dado que el crecimiento y desarrollo cerebral resultan de la correlación entre la genética y las experiencias de interacción con el ambiente, esto "va a permitir un incomparable aprendizaje y el desarrollo de habilidades sociales, emocionales, cognitivas, sensoperceptivas y motoras, que serán la base de toda una vida" (OEA, 2010, p. 7). En concordancia con lo anterior, Campos (2011) afirma que el desarrollo "es un proceso constructivo, con una complejidad única en cada persona, y que tiene como base, indiscutiblemente, los primeros años de vida” ( $\mathrm{p}$. 1). Los anteriores planteamientos se respaldan con los aportes de Pérez, Rizzoli, Alonso y Reyes (2017) al asegurar que los primeros tres años de vida junto con la gestación son muy sensibles. De hecho, se estima que durante dicho periodo "los cerebros de los niños son como una esponja que absorben todas las experiencias e interacciones de su entorno. Cuanto mejores sean las condiciones para el cuidado de la salud, más sinapsis se formarán” (p. 88).

El cerebro experimenta grandes cambios: crece, se desarrolla y pasa por periodos sensibles y críticos para algunos aprendizajes, por lo que "requiere de un entorno con experiencias significativas, estímulos multisensoriales, recursos físicos adecuados; pero, principalmente, necesita de un entorno potenciado por el cuidado, la responsabilidad y el afecto de un adulto comprometido" (OEA, 2010, p. 8).

Entre tantas razones, se puede insistir en que en los primeros años de vida el cerebro humano necesita las experiencias del ambiente para empezar a funcionar adecuadamente. En esta etapa las experiencias ejercen gran influencia en determinadas estructuras y circuitos neuronales, y pasa por un momento conocido como Periodos sensibles; los cuales permiten cierto aprendizaje y corresponden a los periodos "en que es posible incorporar nuevas habilidades. Depende, en parte, de la capacidad de crear nuevas sinapsis y en cerebros exigidos, puede extenderse por mucho tiempo" (Pinto, 2008, p. 20). Según Campos (2014), durante estos periodos, los circuitos neuronales son maleables, plásticos y más receptivos a la estimulación del entorno. En estos periodos, no solamente las experiencias y los estímulos modifican la arquitectura del cerebro, sino que la ausencia de los mismos puede traer consecuencias estructurales y funcionales.

Con relación a los Periodos críticos, estos "permiten el desarrollo de una determinada habilidad, por ejemplo: la visión y audición se desarrollan primordialmente desde el nacimiento hasta los 5 meses de edad. Posteriormente, una catarata congénita o una sordera anatómica no operadas, dejan secuelas 
irrecuperables" (Pinto, 2008, p. 20). El primer año de vida es fundamental para la definición y estimulación de dichos períodos, su fin es lograr un cableado neuronal estable y duradero (Maya y Rivero, 2010, p. 65).

\section{Neurodesarrollo}

El desarrollo a nivel cerebral en los primeros años de vida se encuentra en un proceso continuo de crecimiento, a veces acelerado o demorado de acuerdo con las modificaciones y conexiones propias de la continua estimulación que el entorno provee.

El conocimiento del sistema nervioso nos ayudará a poder comprender los déficits que pueden aparecer por un desarrollo anormal del cerebro o a causa de daños en el mismo a edades tempranas. Dependiendo del momento en el que se produzcan estas anormalidades o daños (durante el embarazo, en el periodo perinatal o en el transcurso de la infancia), sus repercusiones variarán (Ponce, 2017, p. 407).

Está demostrado que el mayor desarrollo del cerebro ocurre durante los tres primeros años y, en parte, depende del ambiente en el que el niño se desenvuelve, de la alimentación, la salud, el cuidado y las posibilidades de interacción que se le brinden. "La atención, el cuidado y una educación de calidad son factores determinantes para que los procesos físicos, sociales, emocionales y cognitivos se desenvuelvan apropiadamente y contribuyan a ampliar las opciones de los niños a lo largo de su vida” (MEN, 2009, p. 8).

Ponce (2017) afirma que el proceso en el cual el niño participa junto a su medio ambiente influyéndose mutuamente y evolucionando en una dirección particular hace parte de su proceso de neurodesarrollo. En este mismo sentido cita a Artigas, Guitart, Gabau, (2013) para referir que "el neurodesarrollo es un proceso evolutivo producto de la adaptación al medio, mediante pautas de comportamiento, en busca del mantenimiento de una tasa reproductiva capaz de sostener la supervivencia de la especie" (p. 408).

Por su parte, Campos (2014) define el neurodesarrollo como "un proceso dinámico, multifacético y multidimensional, relacionado al crecimiento y desarrollo del sistema nervioso central y del cerebro. Es fruto de la interacción entre genética y ambiente, e involucra muchos factores y afecta directamente el comportamiento del ser humano” (p. 40). Sumado a lo anterior, Márquez (2012), dice que el neurodesarrollo hace referencia a la formación del sistema nervioso desde el desarrollo embrionario hasta la edad adulta. El neurodesarrollo o la 
neurología del desarrollo tiene como interés “estudiar en condiciones de salud o enfermedad, los procesos de adaptación del niño durante su interacción con el ambiente" (p. 14); por su parte, Cifuentes (2014) y Figueroa, Campoverde y Calle (2015) agregan que conocer la evolución del neurodesarrollo durante el primer año de vida es crucial para la toma de decisiones frente a estimulación e intervención oportunas.

Dentro del neurodesarrollo existe un proceso llamado neuroplasticidad "que representa la capacidad del sistema nervioso de cambiar su reactividad como resultado de activaciones sucesivas" (Garcés y Suárez, 2014, p. 119). En consonancia con lo anterior, Campos (2014) agrega que la neuroplasticidad es la capacidad del cerebro de modificarse en su organización y funcionalidad ante la experiencia ambiental. Al respecto, Pinto (2008) agrega que "corresponde a la capacidad del cerebro de responder y reorganizarse frente a noxas que lo afecten seriamente y esta condición es propia de la primera década de vida" (p. 20).

Teniendo en cuenta lo anterior, resulta fundamental que en los primeros años de vida se aproveche la neuroplasticidad y se tengan en cuenta todos los elementos que favorecen el cerebro para optimizar el curso del desarrollo; para ello, Romo, Liendo, Vargas, Rizzoli, y Buenrostro (2012) plantean pruebas de tamizaje de neurodesarrollo y Mendoza, Ares y Sáenz (2017) investigan la deteccion precoz de posibles trastornos del neurodesarrollo en los primeros años de vida; con base en ello, afirman que el seguimiento del neurodesarrollo en la primera infancia resulta primordial para evitar posibles trastornos y promover un adecuado desarrollo infantil.

\section{Estimulación adecuada}

Es posible afirmar, que hacer un seguimiento del neurodesarrollo a través de la estimulación, pues esta permite la relación diaria con el bebé, González (2007) afirma que "a través de ésta el niño utilizará al máximo sus capacidades e irá ejerciendo mayor control sobre el mundo que le rodea al tiempo que sentirá gran satisfacción al descubrir que puede hacer las cosas por sí mismo” (p. 19).

La estimulación tiene lugar a través de la repetición útil de diferentes eventos sensoriales que aumentan, por una parte, el control emocional proporcionando al niño una sensación de seguridad y goce, por otra amplían la habilidad mental que le facilita el aprendizaje, ya que se desarrollan destrezas para estimularse a través del juego libre y del ejercicio, de la curiosidad, la exploración y la imaginación (González, 2007, p. 19). 
La estimulación durante el neurodesarrollo cobra valor en la medida que permite la prevención e identificación oportuna de alteraciones en el desarrollo, lo cual es destacado por diversas investigaciones; por ejemplo, Chávez (2003) plantea que la detección temprana de alteraciones en el desarrollo es crucial para dar solución a objetivos clínicos, educativos y sociales, en los que se incluye la necesidad de identificar las causas de dicha desviación. Igualmente, Rosselli, Matute y Ardila (2010), opinan que es indispensable la detección temprana de cualquier alteración en el desarrollo dado que algunas condiciones tienen perfiles neuropsicológicos característicos que se pueden utilizar como indicativos de una disfunción en una región cerebral particular. Por su parte, Portellano (2005) y Fernández (2012), agregan que la prevención de un daño cerebral se ve favorecida por la neuroplasticidad, especialmente en los primeros años de vida, pues tiene la posibilidad de disminuir el riesgo de reacciones psicopatológicas.

En este sentido, Orton et al. (2009 citado en Ortiz, Robayo y Alejo de Paula, 2014) argumentan que las intervenciones y programas de estimulación adecuada tienen grandes beneficios para el desarrollo infantil, obteniendo resultados positivos a nivel cognitivo y motor; Pelayo, Solovieva, Quintanar y Reyes (2014) realizan aportes frente a los efectos de la estimulación del neurodesarrollo afirmando que esta permite disminuir la manifestación de alguna discapacidad que en el futuro pudiera manifestarse. Baxter, Madriz y Mora (2011) afirman el anterior postulado agregando que estimulación y primeros años de vida son un binomio determinante para la prevención de la discapacidad. Baker y López (2013) por su parte, investigan las intervenciones de estimulación en los países en vías de desarrollo y concluyen que los procesos de estimulación de mayor intensidad y duración son los más eficaces, además, para ser más efectivos deben centrarse en los niños más pequeños y buscar activamente la participación de las familias y los cuidadores. Por su parte, García (2014 citado en Ortiz, Robayo y Alejo de Paula, 2014) refiere que la estimulación adecuada es el

Resultado de estudios e investigaciones en diferentes áreas que abarcan el desarrollo infantil, donde luego de tener un entendimiento tanto del desarrollo neurológico, físico y cognitivo del niño, se implementan estrategias que buscan sustentar la información relevante y pertinente para cada etapa del desarrollo, brindando la oportunidad de tener una estructura cerebral y física adecuada en el niño (p. 125).

Se entiende por estimulación adecuada "al proceso a través del cual se busca potencializar las capacidades de desarrollo de un individuo respetando siempre su ritmo de evolución” (González, 2007, p. 21). Ortiz, Robayo y Alejo de Paula (2014) lo definen "como el conjunto de acciones dirigidas a promover las capacidades 
físicas, mentales y sociales del niño, a prevenir el retraso motor y rehabilitar las alteraciones motoras y los déficits sensoriales" (p. 120). En concordancia con lo anterior, Ramírez y Parra (2010) afirman que un medio eficaz para potencializar al máximo las habilidades en el desarrollo de los niños es la estimulación.

Desde la perspectiva de la estimulación adecuada se evidencia la importancia de tener en cuenta el neurodesarrollo, "ya que todo niño nace con la necesidad biológica de aprendery cualquier estimulación que se le brinde durantelos primeros 12 meses, tiene más impacto en su crecimiento cerebral que en cualquier otra etapa de la vida" (González, 2007, p. 19); dentro de los procesos de estimulación, es importante realizar acciones pertinentes para la etapa en la que se encuentre cada niño, brindándole la oportunidad de tener un crecimiento estructural, físico y de habilidades adecuado a su edad y a su ritmo. Todo lo que se debe hacer para un adecuado desarrollo es "proporcionar información visual, táctil, auditiva y motriz, dentro del marco de una relación amorosa, a la velocidad, intensidad y ritmo propio del niño" (Zambrano 2010, citado en Cedeño y Vásquez, 2014, p. 13). Desde diversas disciplinas como la psicología, la pedagogía y la neurofisiología señalan "la importancia de los primeros años de vida para el futuro desarrollo del individuo, dada la plasticidad del sistema nervioso y su acelerada disminución con el transcurso del tiempo de manera que los dos primeros años de vida son cruciales" (Huepp, 2005, p. 7).

\section{Primera infancia}

Estudios relacionados con la estimulación en los primeros años de vida, están generando cambios significativos acerca de la atención y educación de la primera infancia, "en tanto que padres, educadores, organismos gubernamentales y no gubernamentales empiezan a entender que la educación, principalmente en esta etapa de la vida, desempeña un papel casi protagónico en la estructuración y funcionalidad del sistema nervioso y del cerebro" (OEA, 2010, p. 7).

En estudios sobre estimulación, Maslucan, Velayarce, y Rodríguez (2013) concluyen que existe una relación altamente significativa entre el nivel de conocimiento del adulto sobre estimulación y el desarrollo de los niños en la primera infancia. Asi mismo, la labor del docente en la relación con el niño también es resaltada, pues permite ayudarle a madurar su sistema nervioso y a fomentar habilidades como la imaginación y la exploración. Tal como lo resaltan las investigaciones de Barzola, Posligua y Chenche (2017); Sanna (2016) y Ramírez, Patiño, y Gamboa, E. (2014). El rol del docente es fundamental no solo para favorecer el desarrollo sino para prevenir posibles daños. Por su parte, Garza (2014), Flores (2013) y Meza (2011), estudian el impacto de la estimulación en la primera infancia 
y concluyen que esta es de suma importancia en dicha etapa, pues ayuda a los niños a desarrollar la empatía y la seguridad, además, permite la consolidación de funciones y habilidades fundamentales para el desarrollo del ser humano, y reafirma lo expuesto anteriormente.

Otras investigaciones, como la de Quintero, Gallego, Ramírez y Jaramillo (2016) y la de Castillo, Ramírez y Ruíz (2017) resaltan la importancia de la formación de los docentes de primera infancia y afirman que son ellos quienes deben asumir al niño como un ser integral en todas sus dimensiones, de tal manera, que cada acción obedezca a la creación de ambientes llenos de sentido y significado. Por su parte, Ramírez, Patiño y Gamboa (2014) agregan que existe una necesidad por parte de los docentes de conocimiento y formación acerca del desarrollo humano en la infancia y de cómo aplicarlo en una atención integral realmente centrada en los intereses y necesidades de los niños. A su vez afirman que los profesionales que atienden la primera infancia "necesitan comprender la importancia de incluir, en su quehacer pedagógico, los avances en las investigaciones acerca del desarrollo cerebral en los primeros años de vida, debido a la trascendencia de este período" (p. 87).

Realizar procesos de estimulación durante el neurodesarrollo en la primera infancia es importante según los aportes de las investigaciones de Barzola, Posligua y Chenche (2017); Ramírez, Patiño y Gamboa (2014); Ortiz, Robayo y Alejo de Paula (2014); y Garza (2014); porque: brinda la oportunidad de tener una estructura cerebral y física adecuada. Permite la creación de conexiones más complejas, permitiendo la adaptación al medio. Implica la creación de ambientes favorables que respondan a las diferencias individuales de los niños y que vayan más allá de un enfoque asistencialista. Favorece la identificación de alteraciones para intervenir de manera oportuna. Permite el desarrollo e incremento de nuevas conexiones sinápticas y extensiones dendríticas entre las neuronas existentes del sistema nervioso central. Y se obtienen resultados positivos en todas las áreas del desarrollo.

\section{Discusión y conclusiones}

Actualmente se cuenta con una variedad de estudios que permiten analizar la importancia de la estimulación adecuada durante el neurodesarrollo en la primera infancia; sin embargo, pocas investigaciones interrelacionan las categorías trabajadas; así mismo, se encontró que la mayoría de estudios son realizados en el área de la salud o la clínica y pocos son efectuados en el ámbito educativo. 
Según la literatura revisada, se aprecia un cambio significativo en la concepción actual del término desarrollo, el cual permite tener una mirada más amplia de la evolución de los niños teniendo en cuenta la variabilidad de sus avances a través del tiempo y los retrocesos que pudiesen ocurrir; resulta fundamental la noción del concepto como un proceso no lineal sino en constante transformación.

En cuanto al neurodesarrollo, se aprecia que a este, es fundamental tenerlo en cuenta para el adecuado desarrollo de los niños, debido a que determina la arquitectura del cerebro a partir de la interacción entre genética y el ambiente en el que vive el niño; por lo tanto, los estudios resaltan la importancia de tener en cuenta no solo el desarrollo del niño, sino también las interacciones que establece con padres, cuidadores y el entorno inmediato.

En lo que refiere a la estimulación en los primeros años de vida, diversos estudios resaltan los beneficios que esta ofrece para aprovechar al máximo las capacidades de los niños y realizar intervenciones centradas en sus logros, optimizando así, las posibilidades que ofrece el cerebro en términos de plasticidad, periodos sensibles y periodos críticos; es por esto que la estimulación constituye un aspecto importante para el adecuado crecimiento, desarrollo y maduración, puesto que garantiza las interacciones con el exterior en óptimas condiciones.

Con relación a lo anterior, se puede decir que el papel de los adultos en la estimulación durante la primera infancia resulta fundamental, pues proporciona los medios y propicia un entorno adecuado para que ellos realicen una contribución significativa en el desarrollo de los niños; del mismo modo, los adultos son quienes tienen la mayor posibilidad de detectar de manera temprana factores de riesgo que puedan afectar el curso del mismo.

En general, la información revisada permite concluir que, en el desarrollo de los niños se presentan constantes avances y retrocesos, los cuales van a depender de la interacción entre genética y ambiente; ante esto, se demuestra que la estimulación adecuada brinda múltiples ventajas para que este se dé en las mejores condiciones y responda a las necesidades particulares que presenta cada niño desde su proceso de neurodesarrollo, en el que se evidencian periodos críticos y sensibles que pueden ser aprovechados al máximo para potenciar las capacidades de los niños, especialmente en la etapa de la primera infancia. 


\section{Referencias}

Alarcón, A. y Ellies, J. (2007). Teorías del desarrollo texto de apoyo didáctico para la formación del alumno. Universidad de Santiago de Chile.

Alegria, A. (2008). Modelo de Entrega de Servicios de Estimulación Temprana Diseñado Hacia la Universalización de la Atención para Fortalecer el Desarrollo del Potencial Intelectual de Niños de o a 3 años de Edad. Tesis para optar grado de Doctor en Educación. Universidad Nacional de Trujillo. Ávila, A. (2012). Adaptación del cuestionario de madurez neuropsicológica infantil CUMANIN de Portellano. Revista Iberoamericana de Psicología: Ciencia y Tecnología 5(1): 91-99.

Barzola, V.; Posligua, J.; y Chenche, W. (2017). La estimulación infantil para niños de 0 - 4 años. Propuesta: guía para madres comunitarias del sector nor-oeste del Cantón Durán. Revista Científica Dominio de las Ciencias, 3 (3), 990-1019.

Baxter, J.; Madriz, L.; y Mora, L. (2011). Prematuridad y estimulación temprana: ¿un binomio determinante para la prevención de la discapacidad? Revista Innovaciones Educativas, 18, 11- 21.

Baker, H.; y López, F. (2013). Intervenciones de estimulación infantil temprana en los países en vías de desarrollo. Lo que funciona, por qué y para quién. Banco Interamericano de Desarrollo.

Campos, A. (2011). La importancia de la primera infancia desde la mirada de las neurociencias. Abstract de la conferencia presentada en el Encuentro Nacional de Primera Infancia, formación de figuras educativas. Distrito Federal - México.

Campos, A. (2014). Los aportes de la neurociencia a la atención y educación de la primera infancia. Cerebrum Ediciones.

Castillo, R., Ramírez, P., y Ruíz, L. (2017). Necesidades de formación profesional en el ámbito de la primera infancia: Percepción y aportes del estudiantado. Revista Electrónica Educare, 21(1), 1-21.

Cedeño, R.; y Vásquez, M. (2014). Estudio actual de los programas de estimulación temprana desarrollados en los 7 centros de educación especial de la Provincia de Manabí y Propuesta de una Guía de integración sensorial para niños y niña con discapacidad visual. Tesis para obtener el titulo de Magister en Educación Especial. Universidad Politécnica Salesiana.

Cifuentes, D. (2014). Evolución del neurodesarrollo durante el primer año de vida en neonatos sometidos a ventilación mecánica. Tesis para obtener el grado de Maestro en ciencias en Pediatría. Universidad de San Carlos de Guatemala, Facultad de Ciencias Médicas, Escuela de Estudios de Postgrado.

Chávez, R. (2003). Neurodesarrollo neonatal e infantil. México: Editorial Médica Panamericana. 
Chinome, J., Rodríguez, L. y Parra, J. (2017). Implementación y evaluación de un programa de estimulación cognitiva en preescolares rurales. Universidad Pedagógica y Tecnológica de Colombia, Tunja (Colombia). Versión preliminar del documento.

Fernández, R. (2012). Cerebrando el aprendizaje. Recursos teórico-prácticos para conocer y potenciar el órgano del aprendizaje. Buenos Aires: Editorial Bonum.

Figueiras, A., Neves, I., Ríos, V., y Benguigui, Y. (2011). Manual para la vigilancia del desarrollo infantil (o-6 años) en el contexto de AIEPI. Organización Panamericana de la Salud. Pp 57.

Figueroa, M., Campoverde, M., y Calle, S. (2015). Intervención Temprana en Niños con Alteraciones en el Neurodesarrollo Desde la Sala Multisensorial. Un reto en la Academia Ecuatoriana. Latin American Journal Of Computing - Lajc, Vol Ii, No. 3. Pp. 55 - 62.

Flores Aguilar, J., (2013). Efectividad del programa de estimulación temprana en el desarrollo psicomotor en niños de o a 3 años. Revista "Ciencia y Tecnología”, 9(4), 101 - 117.

Francisco, R. (2011). Factores asociados a desarrollo psicomotor anormal en niños de 2 años de edad. Tesis de posgrado para obtener el título en la especialidad de: Medicina familiar. Universidad Veracruzana.

Garcés, M., y Suárez, C. (2014). Neuroplasticidad: aspectos bioquímicos y neurofisiológicos. Rev CES Med, 28(1), 119-132.

Garza, J. (2014). El impacto de la estimulación temprana en la primera infancia: Estudio comparativo entre ambiente escolarizado y ambiente hogar. Universidad de Monterrey.

González, C. (2007). Los programas de estimulación temprana desde la perspectiva del maestro. Liberabit,13,19-27.

Huepp, F. (2005). Estimulación temprana a niños de o a 2 años con factores de riesgo de retraso mental. Tesis en Opción al Grado Científico de Doctor en Ciencias Pedagógicas. Instituto Superior Pedagógico "Frank País García” Santiago de Cuba.

Márquez, J. (2012). Neurodesarrollo y estimulación temprana en pediatría. Confederación nacional de pediatría de México responsable: Dr. Ernesto a. Jiménez Balderas. Pp. 234.

Martínez, J. (2014). Desarrollo infantil: una revisión. Investigaciones Andina, 16(29), 1118-1137.

Martins, J., y Ramallo, M. (2015). Desarrollo infantil: análisis de un nuevo concepto. Rev. Latino-Am. Enfermagem, 23(6),1097-104.

Maslucan, I.; Velayarce, L.; y Rodríguez, Y. (2013). Conocimiento materno sobre estimulación temprana y desarrollo psicomotor del lactante mayor. Revista Científica In Crescendo, 4 (2), 327-336. 
Maya, N., y Rivero, S. (2010). Conocer el cerebro para la excelencia en la educación. Innobasque. Agencia Vasca de la Innovación. Pp. 237.

Mendoza, M.; Ares, S.; y Sáenz, B. (2017). Detección precoz de trastornos del neurodesarrollo en los primeros años de vida en niños con cardiopatías congénitas. Revista Española de Discapacidad, 5 (I), 99-111.

Meza, M.; Gómez, M.; y García, I. (2011). Beneficios de la aplicación de un programa de estimulación sensorial en niños y niñas con edades entre tres y cuatro años de edad, con retraso en el desarrollo psicomotor. Tesis que para obtener el grado de Maestría en Educación con acentuación en Desarrollo Cognitivo. Universidad de monterrey.

Ministerio de Educación Nacional (MEN). (2009). Desarrollo Infantil y Competencias en la Primera Infancia. Documento 10. Santafé de Bogotá.

Ministerio de Educación Nacional (MEN). (2013). Estrategia de atención integral a la primera infancia. Fundamentos políticos técnicos y de gestión. Imprenta Nacional.

Mustard, F., Young, M., y Manrique, M. (2003). ¿Qué es el desarrollo infantil? Memoria del foro "Primera infancia y desarrollo. El desafío de la década". Memorias Foro. Primera infancia y desarrollo. El desafío de la década.

Organización de los Estados Americanos (OEA). (2005). Desarrollo infantil temprano. Retomado desde http:/www.oas.org/udse/dit2/por-que/ investigaciones.aspx

Organización de los Estados Americanos (OEA). (2010). Primera infancia: una mirada desde la Neuroeducación.

Organización Mundial de la Salud (OMS). (2007). Desarrollo de la Primera Infancia: Un Potente Ecualizador. Informe Final para la Comisión sobre los Determinantes Sociales de la Salud de la Organización Mundial de la Salud. HELP centro de investigaciones del Instituto Universitario de Estudios Multidisciplinarios, University of British Columbia.

Ortiz, A., Robayo, V. y Alejo de Paula, L. (2014). Revisión sistemática de las intervenciones para la estimulación en niños con retraso motor de o a 12 meses de edad. Corporación Universitaria Iberoamericana. Bogotá. Mov. cient., (1), 118-130.

Pastor, R., Nashiki, R., y Pérez, M. (2010). Desarrollo y aprendizaje infantil y su observación. Universidad Nacional Autónoma de México. Facultad de Psicología. Maestría en Psicología profesional.

Pelayo, H.; Solovieva, Y.; Quintanar, L.; y Reyes, V. (2014). Efectos de la estimulación del neurodesarrollo en niños con antecedentes de encefalopatía hipóxico isquémica. Pontificia Universidad Javeriana, Cali, Colombia. Pensamiento Psicológico, 12 (1), 11-21. 
Pérez, R., Rizzoli, A., Alonso, A., y Reyes, H. (2017). Avances en el desarrollo de la primera infancia: desde Neuronas para programas a gran escala. Bol Med Hosp Infant Mex., 74 (2),86-97.

Pinto, F. (2008). Lo maravilloso y mágico del neurodesarrollo humano. Revista Chilena de Pediatría, 79 Supl (1),18-20.

Ponce, J. (2017). Atención temprana en niños con trastornos del neurodesarrollo. Propósitos y Representaciones, 5, (1), 403-422.

Posada, A.; Gómez, J.; y Ramírez, H. (2016). El niño sano. Una mirada integral. Editorial Médica Panamericana.

Portellano, J. (2005). Introducción a la neuropsicología. Universidad Complutense de Madrid. Madrid: McGraw Hill.

Quintero, S.; Gallego, A.; Ramírez, L.; y Jaramillo, B. (2016). La formación integral de las maestras para la primera infancia: un reto inaplazable. Artículo de Investigación. Revista del Instituto de Estudios en Educación y del Instituto de Idiomas, 25, 22-33.

Ramírez, J., y Parra, M. (2010). Estimulación Temprana en Niños Menores de 2 Años en la Ciudad de Durango. Rev CONAMED, 15 (supl.1), 30-34.

Ramírez, P., Patiño, V., y Gamboa, E. (2014). La educación temprana para niños y niñas desde nacimiento a los 3 años: Tres perspectivas de análisis. Revista Electrónica Educare, 18(3), 67-90.

Romero, A., y Muñoz, M. (2016). Instrumentos de evaluación de pesquisa de neurodesarrollo en la intervención temprana. Fundación Universitaria Los Libertadores Bogotá, Colombia. Revista Tesis Psicológica, 11(2), 54-71.

Romo, B.; Liendo, S.; Vargas, G.; Rizzoli, A.; y Buenrostro, G. (2012). Pruebas de tamizaje de neurodesarrollo global para niños menores de 5 años de edad validadas en Estados Unidos y Latinoamérica: revisión sistemática y análisis comparativo. Boletín médico del Hospital Infantil de México, 69 (6), $450-462$.

Rosselli, M.; Matute, E.; y Ardila, A. (2010). Neuropsicología del Desarrollo Infantil. Ciudad de México: Editorial El Manual Moderno.

Sanna, V. (2016). Experiencias de Atención Temprana del Desarrollo Infantil. El impacto de una institución en su comunidad (Tesis de posgrado). Bernal, Argentina: Universidad Nacional de Quilmes. Disponible en RIDAA Repositorio Institucional de Acceso Abierto http://ridaa.unq.edu. ar/handle/20.500.11807/237.

Sibaja, J., Sánchez, T. Rojas, M., y Fornaguera, J. (2016). De la neuroplasticidad a las propuestas aplicadas: estimulación temprana y su implementación en Costa Rica. Universidad de Costa Rica. Revista Costarricense de Psicología, 35 (2), 141-159.

Shonkoff , J., y Garner, A. (2012). Los efectos de toda la vida de la adversidad de la primera infancia y el estrés tóxico. Pediatría, 129 (1): e232-46. 